\title{
DIÁLISIS PERITONEAL AGUDA EN PEDIATRÍA
}

\author{
ACUTE PERITONEAL DIALYSIS IN PEDIATRICS
}

Juan José García-Bustinza1,a

\section{RESUMEN}

Objetivos: Fundamentar por qué la diálisis peritoneal sigue siendo en nuestro país la terapia de elección para injuria renal aguda (IRA). Actualizar los conceptos más importantes de diálisis peritoneal, como indicaciones, contraindicaciones, catéter a usar, prescripción médica y complicaciones. Métodos: Se realizó una revisión de bases de datos sobre diálisis peritoneal en la edad pediátrica, lo que permite fortalecer la formación educativa de médicos, pediatras y nefrólogos pediatras en diálisis peritoneal. Resultados: No existen indicaciones absolutas basadas en datos de laboratorio o signos clínicos por lo que la decisión de diálisis debe ser individual. Conclusión: La diálisis peritoneal en pediatría es la terapia de elección en pacientes con diagnóstico de IRA con requerimiento de terapia de reemplazo renal.

Palabras clave: Diálisis peritoneal; Pediatría; Insuficiencia renal aguda. (fuente: DeCS BIREME)

\section{ABSTRACT}

Objective: To establish why peritoneal dialysis remains the therapy of choice for acute renal injury (ARI) in our country. Update the most important concepts of peritoneal dialysis, such as indications, contraindications, catheter to be used, medical prescription and complications. Methods: A review of databases on peritoneal dialysis in the pediatric age was carried out, which allows to strengthen the educational training of pediatric doctors, pediatricians and nephrologists in peritoneal dialysis. Results: There are no absolute indications based on laboratory data or clinical signs, so the dialysis decision must be individual. Conclusions: Peritoneal dialysis in pediatrics is the therapy of choice in patients with ARI who require renal replacement therapy.

Key words: Peritoneal dialysis; Pediatrics; Acute renal failure. (source: MeSH NLM)

\section{INTRODUCCIÓN}

La Injuria Renal Aguda (IRA) no es frecuente en niños, sin embargo la incidencia se está incrementando, a medida que se perfeccionan las modalidades de tratamiento en niños con falla multiorgánica en las unidades de cuidados intensivos pediátricos y neonatales.

La Diálisis Peritoneal Aguda (DPA) sigue siendo la terapia de elección en pacientes con IRA en países en vías de desarrollo, siendo la hemodiálisis reservada para niños de mayor peso. En nuestro país no contamos con técnicas continuas extracorpóreas por su alto costo ${ }^{1,2}$. Entre las ventajas más importantes de la diálisis peritoneal se debe mencionar que no necesita de acceso vascular o cebado de sangre, no requiere heparinización sistemática, es lenta y continua por lo cual hay menor riesgo de inestabilidad hemodinámica. Al ser mayor el área por kg de peso de la membrana peritoneal en niños su eficiencia es mayor que en adultos, los grandes poros de la membrana peritoneal permite el aclaramiento de solutos de alto peso molecular generando una ventaja con respecto a la hemodiálisis en pacientes sépticos ya que permitiría remover citoquinas tóxicas ${ }^{3}$. Entre las desventajas se encuentran que la corrección de parámetros metabólicos es lenta como la acidosis y la hiperkalemia, hay riesgo de peritonitis, no es de elección en pacientes con enfermedad pulmonar severa, errores innatos del metabolismo o procesos intraabdominales.

Las causas más frecuentes de IRA con requerimiento dialítico en países en vías de desarrollo son el Síndrome Uremico Hemolítico, la septicemia, Insuficiencia cardiaca y la necrosis tubular aguda ${ }^{4,5}$. Siendo la IRA con requerimiento dialítico más frecuente en niños pequeños donde la diálisis peritoneal en muchos casos es su única alternativa, se hace necesario tener un adecuado manejo de este tipo de terapia para el beneficio de nuestros pacientes.

\footnotetext{
' Nefrólogo Pediatra, Asistente de la Unidad de Nefrología Pediatrica HNERM.
}

a Facultad de Medicina Alberto Hurtado UPCH. 


\section{CONCEPTOS IMPORTANTES EN DIÁLISIS PERITONEAL}

Peritoneodiálisis:Todas aquellas técnicas de diálisis que utilizan el peritoneo como membrana de diálisis y su capacidad para permitir, tras un periodo de equilibrio, la transferencia de agua y solutos entre la sangre y la solución de diálisis. La estructura anatomofuncional de la membrana peritoneal, las características físicoquímicas de la solución de diálisis y el catéter, constituyen los tres elementos básicos de ésta técnica de diálisis.

Cavidad peritoneal: Espacio comprendido entre el peritoneo parietal y visceral. En condiciones normales contiene unos $10 \mathrm{ml}$ de líquido, con alto contenido en fosfatidilcolina. Esta cavidad puede acumular grandes volúmenes.

Membrana peritoneal: Membrana serosa continua, que se comporta como una membrana semipermeable imperfecta (permite paso de agua y solutos en función de su tamaño). Cubre la superficie de las vísceras abdominales (peritoneo visceral) y la superficie interna de la pared abdominal (peritoneo parietal).

Catéter peritoneal: Es el elemento que permite la comunicación entre la cavidad peritoneal y el exterior; debe permitir el flujo bidireccional de dializado sin molestias ni dificultad.

Línea de transferencia (prolongador): Tubo que conecta el catéter con el sistema de diálisis.

Conector 0 adaptador: Pieza que une el catéter con la línea de transferencia; puede ser de titanio o de plástico.

\section{INDICACIONES DE LA DIÁLISIS PERITONEAL EN NIÑOS}

No existen indicaciones absolutas basadas en datos de laboratorio o signos clínicos por lo que la decisión de diálisis debe ser individual. Sin embargo, existe una lista de condiciones las cuales se muestran como ayuda y sugerencias:

\section{Sobrecarga de líquidos}

- Edema agudo pulmón

- Insuficiencia cardíaca congestiva

- Hipertensión refractaria al tratamiento médico

- Imposibilidad para nutrición adecuada

- Balances positivos persistentes

2. Alteraciones metabólicas y electrolíticas severas no corregibles con tratamiento médico
- Hiperkalemia

- Hiponatremia refractaria

- Hiperfosfatemia

- Acidosis metabólica intratable $(\mathrm{pH}<7,2$, con bicarbonato $<12 \mathrm{mEq} / \mathrm{l}$ )

\section{Compromiso urémico}

- Con síntomas de depresión del sistema nervioso central, pericarditis

\section{Toxinas exógenas}

- Litio, salicilatos, etanol, metanol, aminoglucósidos, teofilina, fenobarbital

\section{CONTRAINDICACIONES DE LA DIÁLISIS PERITONEAL}

1. Onfalocele

2. Gastrosquisis

3. Derivación ventrículo peritoneal

4. Hernia diafragmatica

5. Extrofia vesical

6. Cirugía abdominal reciente

\section{INDICACIÓN DEL CATÉTER}

En cuanto a la colocación del catéter para diálisis peritoneal aguda si bien el procedimiento tradicional es usando el catéter rígido, la Guía Europea para Terapia de Reemplazo Renal en niños con Insuficiencia Renal Aguda recomiendan el uso del catéter Tenchkoff colocado en forma quirúrgica más omentectomía parcial como el acceso de elección ${ }^{4,5}$ por tener menor número de complicaciones que el catéter rígido ${ }^{6}$ y que permite asegurar un sistema herméticamente cerrado y la posibilidad de realizar diálisis automatizada con muy buenos resultados ${ }^{7}$. Wong y col. compararon 64 niños: 33 usaron catéter rígido y 34 Tenckhoff con edades, peso, enfermedad primaria, duración de diálisis y pronóstico similares. Se encontró mayor número de obstrucción de flujo y mayor cantidad de complicaciones y recolocación de catéteres en los que usaron los rígidos. Concluyen que los catéteres Tenckhoff fueron asociados a menor número de complicaciones y es el de elección para diálisis peritoneal aguda.

En los neonatos que por algún motivo no pueda colocarse un catéter Tenchkoff en forma inmediata y con urgencia de diálisis, donde el uso del catéter rígido provoca muchas efusiones, utilizar un catéter venoso central como acceso peritoneal se ha reportado con menor tasa de complicaciones $s^{8,9}$. 


\section{COLOCACIÓN DEL CATÉTER POR CIRUJANO PEDIATRA}

En quirófano y bajo anestesia general, se mide el catéter sobre el abdomen del paciente, intentando determinar donde quedará la punta, los cuff y su sitio de salida, marcándolos si fuera necesario. Luego se practica una incisión abdominal, transversa de 2 o 3 $\mathrm{cm}$. para, supra, o infraumbilical derecha o izquierda.

Divulsión roma del músculo recto. Apertura pequeña del peritoneo para prevenir filtraciones. Una vez abierto el peritoneo se practica una omentectomía generosa para prevenir que este obstruya el catéter.

Exploración digital del abdomen y especialmente del espacio de Douglas donde debe quedar la punta del catéter, espacio de mayor declive para permitir buena entrada y salida de los baños.

Luego se instala el catéter, que puede ser recto o de tipo Swan Neck en su salida y recto o Coil en su punta intraperitoneal.

\section{Colocación de catéter flexible}

- La vejiga se vacía de forma espontánea o mediante cateterizacion si es necesario. La sonda se retira para evitar el riesgo de infección.

- Se verifica permeabilidad de los 3 lúmenes del catéter venoso central de 7 french que se usará como como catéter flexible para diálisis peritoneal aguda con solución salina estéril.

- Se realiza manualmente agujeros adicionales con bisturí en forma distal a la última fenestra del catéter.

- Se prepara el abdomen con solución antiséptica y se cubre con campos estériles.

- Si el paciente esta intubado se indica fentanilo para analgesia, o se instila con xilocaína al 2\% para anestesiar la piel y tejido subcutáneo. Si el paciente está con hipoxemia, edema agudo pulmonar o encefalopático, es mejor asegurar un buen soporte ventilatorio antes de usar la sedación y de esa forma asegurar una buena vía aérea.

- Se realiza con una bránula número 18 punción en la línea media a un centímetro por debajo del ombligo en forma perpendicular hasta la cavidad peritoneal. Instilar solución salina o solución dializante aséptica, aspirar y verificar si se está en cavidad peritoneal para completar hasta $5 \mathrm{cc} / \mathrm{kg}$ de solución.

- Retirar la aguja y dejar la cánula en el peritoneo. Pasar la guía del catéter a través de la cánula. Se debe avanzar libremente, en caso de resistencia retirar y reiniciar el procedimiento nuevamente. Una vez que la guía se encuentre en cavidad peritoneal, retirar la cánula.
- Pasar el dilatador a través de la guía asegurándose que solo pase lo suficiente para dilatar la pared. En caso de resistencia realizar una pequeña incisión de 3 a $4 \mathrm{~mm}$ para facilitar la entrada. Retirar el dilatador y pasar el catéter a través de la guía hasta por lo menos 5 a $7 \mathrm{~cm}$ del último orificio, luego retirar la guía y fijar el catéter a la piel.

- Verificar el retorno por todos los lúmenes y utilizar el de mayor flujo, sellar los otros.

\section{Prescripción de diálisis}

El catéter, el adaptador, el prolongador y las líneas de infusión y drenaje deben de formar un sistema cerrado. Para volúmenes menores de 100 cc existen en el mercado set completos para diálisis peritoneal manual. La cicladora se puede usar con volúmenes mayores a $100 \mathrm{cc}$ aunque con volúmenes pequeños el espacio muerto de las líneas y la inexactitud del drenaje podría disminuir la eficacia del tratamiento.

El sistema calentador es muy importante porque la solución tiene que ingresar tibia a la cavidad peritoneal y es necesaria calentarla si no se dispone de calentador con bolsa de agua caliente o manta térmica, de colocarse frio produce molestias, como dolor, vasoconstricción a nivel del peritoneo y puede haber problemas con la ultrafiltración.

El volumen de infusión al inicio en un paciente con IRA debe ser de $10 \mathrm{cc} / \mathrm{kg}$ en menores de $10 \mathrm{Kg}$ o de 300 $\mathrm{cc} / \mathrm{m} 2$ para mayores de $10 \mathrm{Kg}$ y luego subir en forma progresiva hasta $30 \mathrm{cc} / \mathrm{kg}$ o $800 \mathrm{cc} / \mathrm{m} 2$ tratando de no infundir más volumen por el riesgo de efusión 10 y evitar una presión abdominal mayor de $12 \mathrm{~cm}$ de agua por estar relacionada en pacientes críticos con mortalidad y además con excesiva cantidad de líquido, dolor y $\mathrm{pH}$ ácido de la solución, realizando recambios cortos en niños grandes puede ser en promedio de una hora pero en lactantes se acorta a 20 a 30 minutos ${ }^{11,12}$.

Debe añadirse heparina 250-500 UI/Litro durante las primeras 48 horas si el líquido es hemático, para evitar la formación de fibrina en el interior del catéter y mejorar la permeabilidad de la membrana peritoneal a los solutos. Si bien la heparina no parece difundir a través del peritoneo se debe usar con precaución en neonatos y en pacientes con alteración en la coagulación ya que en dichos casos y con recambios rápidos la heparina podría ser absorbida.

Se debe realizar control de electrolitos en forma frecuente ya que si el potasio sérico disminuye a menos de $5.5 \mathrm{mEq} / \mathrm{lt}$ se tiene que añadir 3 a $4 \mathrm{mEq} / \mathrm{L}$ de potasio a la solución de diálisis. En recién nacidos y lactantes pequeños se debe monitorizar la glucosa por el riesgo de hiperglicemia asociada a soluciones de dializado de altas concentraciones de dextrosa. 


\section{Profilaxis}

Para reducir la incidencia de peritonitis se debería usar profilaxis antibiótica con una cefalosporina de primera generación una hora antes de la inserción del catéter de diálisis peritoneal.

Si bien la Vancomicina profiláctica también ha demostrado ser efectiva, su uso rutinario no se recomienda por el potencial desarrollo de microorganismos resistentes.

La profilaxis intravenosa preoperatoria reduce la peritonitis temprana pero no la infección del orificio de salida o el túnel subcutáneo.

\section{COMPLICACIONES}

\section{Obstrucción del catéter}

El drenaje inadecuado puede producirse por malposición o acodamiento del catéter, obstrucción de la luz por fibrina, peritonitis u oclusión de los agujeros laterales por el epiplón. Los catéteres temporales se han asociado a mayor riesgo de fugas y mal funcionamiento. La adición de heparina puede prevenir la formación de coágulos de fibrina, con mínima absorción a la circulación sistémica. La omentectomía parcial en forma rutinaria disminuye considerablemente esta complicación.

\section{Fugas}

Producen mal funcionamiento de la diálisis y pueden ocurrir hacia el punto de inserción, como consecuencia de infección de la puerta de entrada o excesiva apertura de la pared abdominal en la inserción del catéter, o por volúmenes altos de solución de dializado. Las fugas pueden observarse también en el celular subcutáneo, alrededor de una incisión quirúrgica previa. La suspensión temporal de la diálisis es con frecuencia suficiente para resolver el problema.

\section{Infecciones}

Es relativamente frecuente en diálisis prolongada, puede presentarse como líquido turbio, signos de irritación peritoneal con fiebre y dolor abdominal o de forma más sutil como aumento de reactantes de fase aguda o deterioro clínico del paciente. Se recomienda realizar ante la mínima sospecha un estudio citológico del líquido peritoneal e iniciar antibióticos si el recuento leucocitario es mayor a 100 células/cc con más de $50 \%$ de neutrófilos tras 2 horas de permanencia. Si se cuenta con cefepime podría iniciarse como monoterapia. Si no está disponible los gérmenes Gram positivos deben cubrirse con vancomicina y los Gram negativos con ceftazidima o aminoglicósidos ${ }^{12}$. Siendo la vía de elección la intraperitoneal a la endovenosa, aunque se debe considerar la tolerabilidad de algunos antibióticos como la vancomicina intaperitoneal. Se suspende uno de los 2 antibióticos apenas se obtenga el Gram o el cultivo. El tratamiento es de 2 semanas aún con cultivo negativo y si es Staphylocococo aureus, Pseudomonas, anaerobios o infecciones polimicrobianas donde el tratamiento es de 3 semanas.

Lasindicaciones de retiro de catéter porcomplicaciones infecciosas son:

- Peritonitis refractaria (persistencia tras 5 días de tratamiento antibiótico adecuado)

- Peritonitis recurrente (en las 4 semanas posteriores al tratamiento)

- Infecciones del orificio refractarias

- Peritonitis fúngica

- Considerar en micobacterias, pseudomonas, múltiples organismos entéricos y peritonitis recidivante

\section{Peritonitis eosinofílica}

Se diagnostica cuando los eosinófilos representan más del $10 \%$ del total de leucocitos del dializado, suele suceder en un paciente asintomático con líquido efluente turbio. Puede tratarse de una reacción alérgica local a componentes del líquido de diálisis, o a sustancias liberadas del equipo de diálisis. Es típicamente autolimitada.

\section{Complicaciones metabólicas}

Relacionadas con el empleo de soluciones glucosadas que pueden provocar hiperglicemia e hiperlipemia. A mediano o largo plazo pueden provocar desnutrición por pérdida de aminoácidos con el dializado. En casos de hiperglicemias severas puede añadirse Insulina al líquido de diálisis, retirándola en los últimos 2 a 3 recambios para evitar el riesgo de hipoglicemia post diálisis. Añadir $1.0 \mathrm{UI}$ de Insulina por cada 5 gramos de glucosa en solución de dializado.

\section{Otras complicaciones}

- Asociadas al aumento de la presión intaperitoneal: hernia abdominal, fundamentalmente inguinal, hidrocele en recién nacidos, efusión subcutánea e hidrotórax.

- Asociadas a la técnica de inserción o retiro del catéter: hemoperitoneo, quiloperitoneo, perforación de la vejiga, colon o extrusión del epiplón.

- Esclerosis peritoneal en casos de peritonitis a repetición y fibrosis peritoneal, derivada de la glicosilación no enzimática producida por la glucosa hipertónica de las soluciones de diálisis. 


\section{Suspensión de la diálisis peritoneal}

Se indica la suspensión de la diálisis peritoneal cuando se observa recuperación de la función renal en forma sostenida, con diuresis adecuada y disminución progresiva de la urea y otros metabolitos de desecho.

\section{CONCLUSIÓN}

La diálisis peritoneal en pediatría es un procedimiento seguro, simple y barato en niños con falla renal aguda con requerimiento de terapia de reemplazo renal y con una eficiencia igual o mayor que otras alternativas más caras y complejas como la hemodiálisis y la hemodiafiltración.
Financiamiento: Autofinanciado.

Conflicto de interés: El autor declara no tener conflicto de interés en la publicación de este artículo.

Recibido: 15 de febrero del 2017

Aprobado: 30 de marzo del 2017

\section{REFERENCIAS BIBLIOGRÁFICAS}

1. Verrina E, Cappelli V, Perfumo F. Selection of modalities, prescription, and technical issues in children on peritoneal dialysis. Pediatr Nephrol 2009;24:1453-64.

2. Watson AR, Gartland C. Guidelines by an ad hoc. European committee for elective chronic peritoneal dialysis in pediatric patients. Perit Dial Int 2001 $21: 240-244$.

3. Vasudevan, A., Phadke, K., \& Yap, H. K. (2016). Peritoneal dialysis for the management of pediatric patients with acute kidney injury. Pediatric Nephrology, 1-12.

4. Strazdins V, Watson AF, Harvey B. Renal replacement therapy for acute renal failure in children: European guidelines. Pediatr Nephrol 2004;19:199_ 207.

5. Pumford N, Cassey J, Uttley WS. Omentectomy with peritoneal catheter placement in acute renal failure. Nephron 1994; 68:327-328.

6. Chadha V, Warady BA, Blowey DL, Simckes AM, Alon US. Tenckhoff catheters prove superior to Cook catheters in pediatric acute peritoneal dialysis. Am J Kidney Dis 2000; 35:1111-1116.
7. Golej J, Kitzmuller E, Hermon M, Boigner H, Burda G, Trittenwein G.Lowvolume peritoneal dialysis in 116 neonatal and paediatric critical care patients. Eur J Paediatr 2002; 161:385-389.

8. Cavagnaro F, Mufioz J, Rodríguez J y col. Dialisis peritoneal aguda automatizada en el manejo del síndrome hemolítico urémico. Rev. Chil. Pediatr. 1997; 68 (4):171-174.

9. Yu JE, Park MS, Pai KS. Acute peritoneal dialysis in very low birth weight neonates using a vascular catheter. Pediatr Nephrol 2010; 25(2):367-71.

10. Kohli HS, Bhalla D, Sud K, Jha V, Gupta KL, Sakhuja V. Acute peritoneal dialysis in neonates:comparison of two types of peritoneal access. Pediatr Nephrol 1999;13:241-4.

11. Melvin Bonilla-Félix. Peritoneal dialysis in the pediatric intensive care unit setting. Perit Dial Int 2009; 29(S2):S183-S185.

12. Warady, B. A., Bakkaloglu, S., Newland, J., Cantwell, M., Verrina, E., et al. (2012). Consensus guidelines for the prevention and treatment of catheter-related infections and peritonitis in pediatric patients receiving peritoneal dialysis: 2012 update. Peritoneal Dialysis International, 32(Supplement 2), S32-S86.

Consulte la Versión Electrónica de la Revista:

Facultad de Medicina Humana Universidad Ricardo Palma

http://revistas.urp.edu.pe/index.php/RFMH<smiles>[C+]1=CC=CC1</smiles>

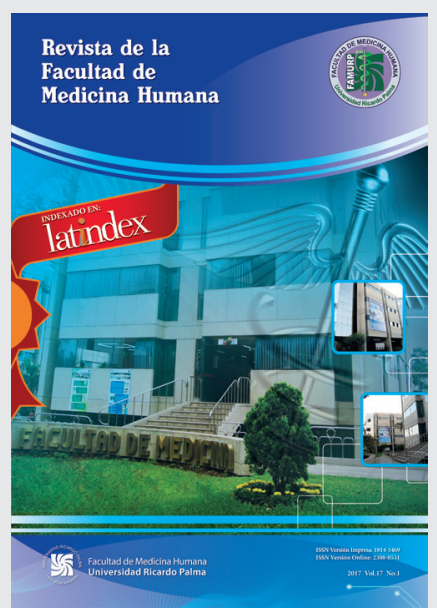

